\title{
Preparation of Carbohydrate Derivatives with PCDA Tails: Applicationfor Cell Surface Recognition
}

\author{
Jae-Ho Jeon, Eun-Hee Jang, Jin-Seon Chюi, Yong-Ill Lee, and Chuljin Ahn*
}

\author{
Department of Chemistry: Changwon National Lnwersity: Changwon 641-773, Korea. ${ }^{*}$-mail: cjahnachangwon.ac.kr \\ Received December 31, 2008. Accepted April 9, 2009
}

Key Words: PCDA amide. Diacetỵlene. Biosensor

Modifications of carbolydrates are found in nature as constituents of many stnictures by biological activity and recognition. Sugar rings have been systematically varied to probe binding sites of carbohydrate-specific proteins. such as enzymes or lectins, which are important for its recognition. ${ }^{3}$

The functionalization at the anomeric center of a carbohydrates can serve as sensor in biological sytem.

Polyacetylene based sensor systems are unique because of blue to red color transitions due to their polymerized diacetylene unit. ${ }^{2.11}$ It is well known that the spacially aligned monomeric diacetylene moieties undergo photo polỳmerization process via a 1,4-addition mechanism and form conjugated chains that give the molecule a significant color change. Due to this unique color change. efforts have been devoted to develop efficient sensor systems based on this polyacetylenes. ${ }^{1 \text { - }}$ "14 $O u r$ plan is devising PCDA (10.12-pentacoasdiy noic acid) biosensor, which is tagging carbohydrates with PCDA dye (Figure 1).

Commercially available $\beta$-D-glucose pentaacetate (1) was transformed into corresponding $\beta$-azide 2 by the known method. ${ }^{15} \beta$-D-glucose pentaacetate azide 2 is reduced with p-toluen sulfonic acid ( $\mathrm{l} .2$ equiv.) in $\mathrm{CH}_{3} \mathrm{OH} / \mathrm{CH}_{2} \mathrm{Cl}_{2}$ as cosolvent system to get carbohydrate $p$-TSA ammonium salt 3 in $90 \%$ yield. The crude 10.12 -pentacosadiynoyl chloride which was prepared from the PCDA and oxalyl chloride was react with carbohydrate $p$-TSA ammonium salt 3 in situ to get glucose derivative + with PCDA tailed in $60 \%$ yield (Scheme 1). ${ }^{16}$

Sinularly, galactose-PCDA anide 8 was prepared from commercially available $\beta$-D-galactose pentaacetate (5). $\beta$ $D$-glucose pentaacetate azide 6 is reduced with $p$-toluen sulfonic acid ( 1.2 equiv.) in $\mathrm{CH}_{3} \mathrm{OH} / \mathrm{CH}_{2} \mathrm{Cl}_{2}$ as co-solvent system to get carbohydrate $p$-TSA anmonium salt 7 in $85 \%$ yield. Treatment of $p$-TSA ammonium salt $7 w$ ith the crude 12-pentacosadiynoyl clloride which is prepared from PCDA and oxalyl chloride give the PCDA connecting galactose derivative 8 in $55 \%$ yield (Scheme 2 ). ${ }^{16}$

In summary. we have prepared precursors of dye labeled carbohydrate ligands which will be tested as a new biosensor. Further studies of biosensing living cell systems such as Concanavalin $\mathrm{A}^{17}$ or tumor cells are now on going and will be discussed soon.

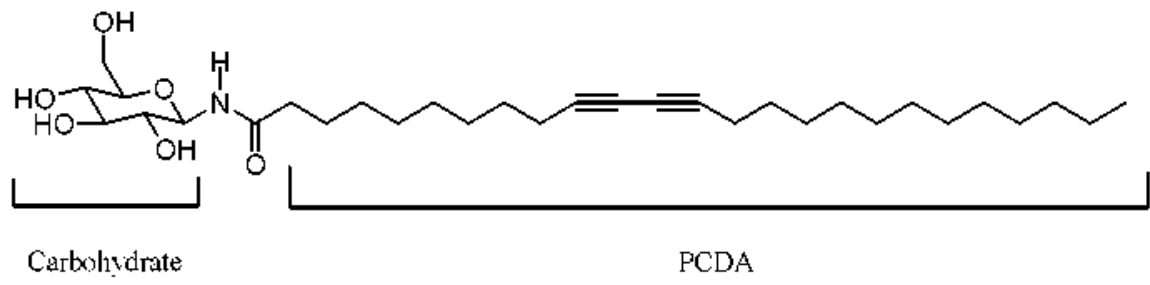

Figure 1
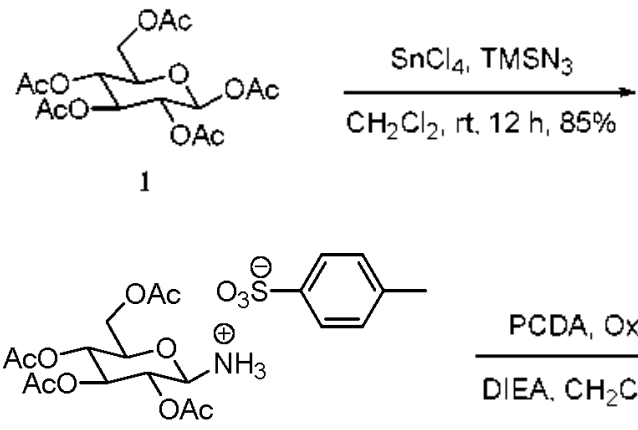

3

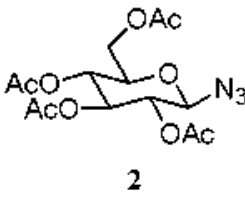

$\frac{p-\mathrm{TsOH}, \mathrm{H}_{2}, 10 \% \mathrm{Pd} / \mathrm{C}}{\mathrm{MeOH} / \mathrm{CH}_{2} \mathrm{Cl}_{2}, \mathrm{rt}, 2 \mathrm{~atm}}$ $\underset{\text { DIEA, } \mathrm{CH}_{2} \mathrm{Cl}_{2}, \text { rt. } 12 \mathrm{~h}, 60 \%}{\stackrel{\text { PCDA, Oxalyl chloride }}{\longrightarrow}}$

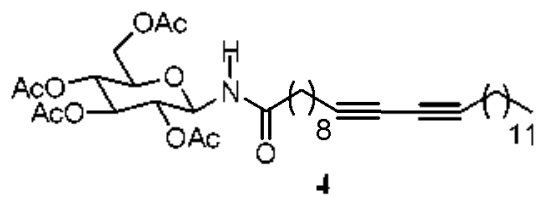

Scheme 1 
<smiles>CC(=O)OCC1(COC(C)=O)COC(=O)OC1OC(C)=O</smiles>

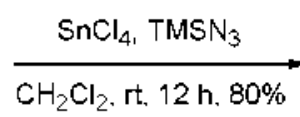

$\mathrm{CH}_{2} \mathrm{Cl}_{2}$, rt. 12 h, $80 \%$

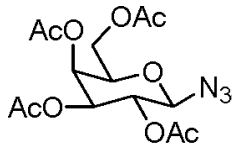

$$
\begin{aligned}
& \underset{\mathrm{peOHOH}, \mathrm{H}_{2}, 10 \% \mathrm{Pd} / \mathrm{C}}{\longrightarrow} \\
& 6 h, 85 \%
\end{aligned}
$$

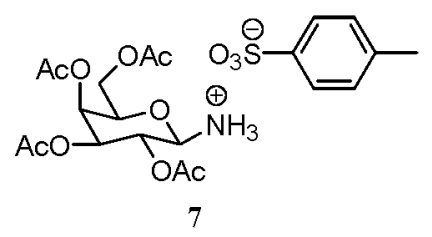

PCDA, Oxalyl chloride

DIEA, $\mathrm{CH}_{2} \mathrm{Cl}_{2}$, rt. $12 \mathrm{~h}, 55 \%$

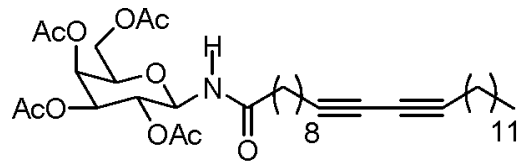

8

Scheme 2

\section{Experimental Section}

General procedure for preparation of $\beta$-D-glucose pentaacetate-PCDA amide 4 and $\beta$-D-galac tose pentancetate-PCDA amide 8. To the solution of PCDA(10.12-pentacosadiynoic acid) $(10.0 \mathrm{mmol})$ in $70 \mathrm{~mL}$ of dried $\mathrm{CH}_{2} \mathrm{Cl}_{-}$. oxalyl chloride (25.2 mmol) was slowly added at room temperature in nitrogen atmosphere. The resulting solution was stirred at room temperature for $1 \mathrm{~h}$. To the solution was added catalytic amount of DMF and stimed for additional $1 \mathrm{~h}$. After concentrating $i n$ vacuo, the residue was redissolved in $60 \mathrm{~mL}$ of $\mathrm{CH}_{2} \mathrm{Cl}_{2}$. The resulting solution was added dropwise to the each solutions containing ammonium p-TSA salts 3 and 4 ( 12.0 mmole) and $N$. -disopropy lethylamine (83.9 mmole) in $100 \mathrm{~mL}$ of anhydrous THF. Each resulting mixture was stirred for $12 \mathrm{~h}$ at room temperature. After checking TLC. each reaction mixture was poured into $500 \mathrm{~mL}$ separatory funnel with washing $200 \mathrm{~mL}$ $\mathrm{CH}_{2} \mathrm{Cl}_{2}$. The organic phase was successively washed by $5 \%$ $\mathrm{HCl}$. saturated $\mathrm{NaHCO}_{3}$, brine and the combined organic fractions were dried over anhydrous $\mathrm{Na}_{2} \mathrm{SO}_{4}$ and concentrated under reduced pressure. Silica gel flash column chromatography (Hexane/Ethyl Acetate 1/1) gave glucose-PCDA amide 4 and galactose-PCDA amide 8 as well.

B-D-Gucose pentancetate-PCDA amide 4: purple solid (yield $60 \%$ ); mp $49-52^{\circ} \mathrm{C}$ (dec.) FT-IR ( $\mathrm{ZnS}$ window); 2294.1 , $2853.5 .1747 .0,1675.0 .1531 .7 .1223 .2 .1040 .7 . \mathrm{cm}^{-1} .[\alpha]_{\mathrm{D}}^{15}=$ +18.40 (c $\left.0.01 . \mathrm{CH}_{2} \mathrm{Cl}_{2}\right)$. ${ }^{\mathrm{l}} \mathrm{H} \mathrm{NMR}\left(400 \mathrm{MHz}, \mathrm{CDCl}_{3}\right) \delta 6.35$ (d. $J=9.4 \mathrm{~Hz}, \mathrm{IH}) .5 .30$ (m. $2 \mathrm{H}$ ). 5.07 (dd. $J=10.3 .9 .8 \mathrm{~Hz}$. lH). $4.93(\mathrm{t} . J=9.6 \mathrm{~Hz}, \mathrm{lH}) .4 .33(\mathrm{dd} J=12.7,4.2 \mathrm{~Hz}, \mathrm{lH}) .4 .07$ $($ dd. $J=12.7 .1 .7 \mathrm{~Hz}, 1 \mathrm{H}), 3.85(\mathrm{~m}, 1 \mathrm{H}), 2.24(\mathrm{t}, J=7.0 \mathrm{~Hz} .3 \mathrm{H})$, 2.09 (s. $3 \mathrm{H}) .2 .05(\mathrm{~s} .3 \mathrm{H}) .2 .04(\mathrm{~s} .3 \mathrm{H}) .2 .03(\mathrm{~s} .3 \mathrm{H}) 1.4-1.1(\mathrm{~m}$. $36 \mathrm{H}), 0.87(\mathrm{t}, J=7.0 \mathrm{~Hz} .3 \mathrm{H}),{ }^{13} \mathrm{C} \mathrm{NMR}\left(100 \mathrm{MHz} . \mathrm{CDCl}_{3}\right) \delta$ $173.37 .170 .92,170.58,169.81,169.53,77.95 .77 .52 .77 .26$. 735.03. 61.49. 36.49. 31.82, 29.55, 29.53. 29.51. 29.39. $29.26,29.02 .29 .00,28.94 .28 .80 .28 .75,28.65 .28 .22,28.16$, $25.00,22.60 .20 .68,20.60,20.52$. 19.08, 19.05. 14.07 .

B-D-Galactose pentaacetate-PCDA amide 8: colorless oil (yield 55\%): $\mathbb{R}$ (ZnS window): 2925.6. 2835.8 . 1750.6. 1700.4. 1533.9. 1277.8 $\mathrm{cm}^{-1} .[\alpha]_{D}^{18}=+21.15\left(c 00193 . \mathrm{CH}_{2} \mathrm{Cl}_{2}\right) .{ }^{1} \mathrm{H}$ $\mathrm{NMR}\left(400 \mathrm{MHz}, \mathrm{CDCl}_{3}\right) \hat{o} 6.37(\mathrm{~d} J=9.5 \mathrm{~Hz}, \mathrm{HH}) .5 .45(\mathrm{~d} . J=$ $2.4 \mathrm{~Hz}, \mathrm{lH}) .5 .28(\mathrm{t} . J=9.2 \mathrm{~Hz}, \mathrm{lH}) .5 .13(\mathrm{~m} .2 \mathrm{H}) .4 .08(\mathrm{~m}$. $3 \mathrm{H}), 2.22$ (t. $J=7.0 \mathrm{~Hz} .3 \mathrm{H}) .2 .16(\mathrm{~s} .3 \mathrm{H}) .2 .06(\mathrm{~s} .3 \mathrm{H}) .2 .05(\mathrm{~s}$. $3 \mathrm{H}) .2 .00(\mathrm{~s} .3 \mathrm{H}), 1.7-1.2(\mathrm{~m} .36 \mathrm{H}), 0.89(\mathrm{t} . J=7.0 \mathrm{~Hz} .3 \mathrm{H})$.
${ }^{13} \mathrm{C} \mathrm{NMR}\left(100 \mathrm{MHz} . \mathrm{CDCl}_{3}\right)$ ô 173.03, 170.92. 170.09. 169.73. $169.48,77.98,77.25,76.51,71.88,70.46,67.90,66.79 .64 .81$. $60.77,36.26,31.58 .29 .31,29.29 .29 .27,29.15,29.02 .28 .80$, $28.77,28.71,28.57,28.51,28.41,27.99,27.82,24.80 .22 .37$, $20.46,20.39 .20 .31,20.25,18.84,18.81,13.83$.

Acknowledgments. This work is supported by the Korea Research Grant funded by the Korean Governument (MOEHRD. Basic Research Promotion Fund) (KRF-2007-412-J0093).

\section{References and Notes}

1. Lindhorst, T. K. Essentials of Carbohydrate Chemistry and Biochemistry; Wiley-VCH Verlag Gmbh, D 69469 Weinheim: Weinheim, New York, Chichester, Brisbane, Singapore, Toronto 177-183.

2. Charych, D. H. Nagy, J. O.; Spevak, W. Bednarski, M. D. Science 1993, 261, 585-588.

3. Reichert, A.: Nagy, T. O.: Spevak, W. Charych, D. J.Am. Chem. Soc. 1995, 117, 829-830.

4. Panl, T. T.; Charych, D. Langmitir 1997, 1365-1367.

5. Ma, Z.; Li, T.; Cao, J.; Zou, Z; Tu, T.; Tiang, L. J. Ant Chent. Soc. $1998,120,12678-12679$.

6. Kolusheva, S.; Kafri, R.: Katz, M.; Telinek, R. J. Am Chem. Soc. $2001,123,417-422$.

7. Kolusheva, S.; Shahal, T; Jelinek, R. J. Am. Chent. Soc. 2000, $122,776-780$.

8. Kolusheva, S.: Shahal, T:; Telinek, R. Biochemistry 2000, 39 , 15851-15859.

9. Okada, S. Y; Telinek, R:; Charych, D. Angew. Chent. Int. Ed., $1999,38,29-33$.

10. Su, Y. L.: Li, I. R.: Jiang, L. Colloids Suf. B. Biomterfaces 2004, $38,29-33$.

11. Su, Y. L.; Li, J. R.: Jiang, L. J. Colloid Intelface Sci, 2005, 284 , 114-119.

12. Ma, G.; Cheng, Q. Langmitir 2005, 21, 6123-6126

13. Su. Y.-L. J. Colloid and Interface Science 2005. 292. 271-276.

14. Jung. Y. K.: Park. H. G.; Kim J.-M. Biosensons and Bioelectronics, 2006, 21, 1536-1544.

15. Commercially available from many commercial sources. Also see Sabesan, S.; Neira, S. Cabohidrate research 1992, 223. 169-185.

16. We followed published procedure of preparing analogous $P C D A$ aryl amides; see Aln, D. J.; Chae, E.-H.; Lee, G. S.; Shim, H.-Y; Chang. T.-E.: Ahn, K.-D.: Kim, J.-M. J. Am. Chem. Soc. 2003, $125,8976-8977$.

17. Concanavalin $A$ is the first commercialized lectin protein. It reacts with specific teminal sugar residues and has been used as a useful tool in study ing carbohydrates of cell surfaces. 